\title{
Elaboração de protocolos assistenciais à saúde como estratégia para promover a segurança do paciente
}

\section{Elaboration of health care protocols as a strategy to promote patient safety}

\author{
Antonio Neudimar Bastos Costa \\ Farmacêutico, Gerente de Risco da Santa Casa de Misericórdia de Sobral, Ceará. E-mail: neudimar92@stacasa.com.br \\ Elaine Cristina Bezerra Almeida \\ Enfermeira da Comissão de Controle de Infecção Hospitalar da Santa Casa de Misericórdia de Sobral, Ceará. E-mail: elainecrisbezerra@ hotmail.com \\ Tiago Sousa de Melo \\ Farmacêutico, Professor doutor em biotecnologia do Centro Universitário UNINTA, Sobral, Ceará. E-mail: tiagosousam@gmail.com
}

Resumo: Objetivou-se relatar a experiência vivenciada na elaboração dos procedimentos operacionais padrão em um hospital da zona norte do Estado do Ceará. Trata-se de um estudo descritivo do tipo relato de experiência que discute a importância da elaboração de protocolos para promover a segurança do paciente. Os profissionais envolvidos elaboraram 35 protocolos, os quais abordavam procedimentos de enfermagem, farmácia, nutrição, fisioterapia e atendimento ao público. A principal dificuldade foi reunir o grupo de profissionais e gestores do Hospital para a discussão e validação. Dentre os novos protocolos estão o de coleta de sangue, administração de nutrição enteral e transporte de pacientes adultos.

Palavras-chave: Protocolos clínicos; Qualidade da Assistência à Saúde; Educação em saúde.

\begin{abstract}
This study aimed to report the experience in the elaboration of standard operating procedures at a hospital from north zone of the state of Ceará. It is a descriptive experience report that discusses the importance of developing protocols to promote patient safety. The professionals involved developed 35 protocols which adressednursering, pharmacy, nutrition, phisioterapy and public attendance procedures. The main difficulty was to gather the group of professionals and managers from the hospital for the discussion and validation.Among the new protocols are protocols of blood collection, enteral nutrition administration and adult patient transport.
\end{abstract}

Keywords: Clinical Protocols; Quality of Health Care; Health Education. 


\section{INTRODUÇÃO}

Sabe-se que após a publicação do livro "Errar é humano", em novembro de 1999, pelo Instituto de Medicina dos Estados Unidos da América, várias foram às discussões em torno da qualidade e segurança do paciente, principalmente em países desenvolvidos (VINCENT; AMALBERTI, 2016).

No ambiente hospitalar, a segurança do paciente tem gerado debates por todo mundo e tem recebido várias interpretações, entre elas, a mais conhecida é que segurança do paciente consiste na redução do risco e danos desnecessários associados à assistência em saúde até um mínimo aceitável (CHARLES, 2010).

Segundo Silva e Camerini (2012), o mínimo aceitável se refere àquilo que é viável diante do conhecimento atual, dos recursos disponíveis e do contexto em que a assistência foi realizada, frente ao risco de não-tratamento ou outro tratamento.

Wachter (2013) sugere que a segurança do paciente deve ser valorizada, mais do que nunca, principalmente nos hospitais, onde os profissionais trabalham em busca do melhor para os pacientes, mas que, devido à elevada complexidade, estes podem estar em risco, só pelo fato de lá se encontrarem. Nos hospitais, a chance do erro acontecer é elevada pelo fato de que a prestação de serviços está ligada às complexas interações entre pessoas, instalações, equipamentos e medicamentos.

Um estudo realizado por Aranaz-Andrés et al. (2011), envolvendo 58 hospitais localizados na Argentina, Colômbia, Costa Rica, México e Peru, buscou-se informações sobre a ocorrência de danos ao paciente em países em desenvolvimento ou em transição. Este estudo constatou que de um total de 11.379 pacientes internados, 1.191 sofreram algum tipo de dano, entre eles, $6 \%$ foram associados à morte do paciente e $60 \%$ foram considerados evitáveis.

No Brasil, a avaliação da incidência de danos foi realizada por Mendes et al. (2009), em três hospitais no Rio de Janeiro. O estudo mostrou que os danos mais frequentes foram as infecções associadas aos cuidados da saúde, representando 24,6\% dos danos evitáveis.

A pesquisa sugere que esses eventos evitáveis representam um sério problema para o cuidado hospitalar no Brasil, e que este cenário pode ser modificado por ações que não requerem demasiada complexidade tecnológica.

Com isso, para tentar minimizar ou evitar a ocorrência de danos ao paciente, problemas legais e éticos aos profissionais e descrédito do estabelecimento de saúde pela sociedade é necessário a construção de procedimentos e protocolos assistenciais em saúde, devendo atender aos princípios éticos e legais de cada categoria profissional, considerando-se os preceitos da prática baseada em evidências, às normas e regulamentos do Sistema Único de Saúde, em suas três esferas de gestão e da instituição onde será utilizado (PIMENTA et al., 2015).

Os Procedimentos Operacionais Padrão (POPs) são instruções detalhadas descritas para alcançar a uniformidade na execução de uma função específica (BARBOSA et al., 2011) e são designados para guiar a tomada de decisão, com relação a diagnóstico, gestão e tratamento de situações clinicas específicas (JOUVETL et al., 2011).

Alguns exemplos de processos comumente vistos, no cuidado de saúde, voltadas para a qualidade da assistência são: a higienização das mãos, verificação rotineira da identificação do paciente, não prescrição de medicamentos perigosos sem autorização para fazê-lo, realização de cirurgia segura, intervenções na prevenção de quedas e a adesão dos profissionais aos procedimentos operacionais padrão (VINCENT; AMALBERTI, 2016).

Tradicionalmente esses protocolos operacionais são a base para uma organização segura, estão baseados em evidências científicas e têm como foco a padronização de condutas clínicas e cirúrgicas em ambientes ambulatoriais e hospitalares (WERNECK, 2009).

Os POPs devem descrever também a forma de validação pelos pares, estratégias de implementação, a construção dos desfechos ou resultados esperados e seu uso tende a aprimorar a assistência, favorecer o uso de práticas cientificamente sustentadas, minimizar a variabilidade das informações e condutas entre os membros da equipe de saúde, estabelecer limites de ação e cooperação entre os diversos profissionais (BROZEK et al., 2009).

Esses protocolos devem ser cuidadosamente elaborados e, por isso, é indispensável o envolvimento dos responsáveis pela execução das tarefas, assim como a análise de cada passo a fím de verificar qual é o mais fácil e eficiente a ser seguido (DAINESI; NUNES, 2007).

Pereira et al. (2010) enfatiza que os profissionais responsáveis pela elaboração dos documentos descritivos deverão conhecer as diretrizes clínicas de âmbito nacional e internacional da especialidade em questão.

Sendo assim, para a elaboração de procedimentos padrão faz-se necessário ter um objetivo claro, as metas devem levar em consideração os fatores políticos, sociais, econômicos, culturais e biológicos e ter, primordialmente, o paciente no centro das atenções (CHARLES, 2010).

Além disso, cada uma das etapas de elaboração do POP (Procedimento Operacional Padrão) deverá ter a participação da equipe envolvida, que irá avaliar e validar seus procedimentos, e, se necessário, contratar pessoal especializado para esta função. Nesses casos, é importante que a equipe detenha o conhecimento do setor e interaja com o grupo, conhecendo cada um dos seus processos e discutindo cada novo POP elaborado (BARBOSA et al., 2011).

O presente trabalho teve como objetivo relatar a experiência adquirida durante a elaboração dos Procedimentos Operacionais Padrão, referentes a procedimentos em enfermagem, farmácia, nutrição, fisioterapia e atendimento ao público, por um hospital de referência da zona norte do Estado do Ceará.

\section{MATERIAL E MÉTODOS}

Trata-se de um estudo descritivo do tipo relato de experiência. Representa uma experiência obtida entre os meses de Junho a Setembro de 2017 realizado em um hospital de referência da região Norte do Estado do Ceará. 
Cientes de que o Procedimento Operacional Padrão (POP) deve ser escrito de forma detalhada para a obtenção da uniformidade, seja na produção ou na prestação de um serviço e a fim de otimizar o processo de elaboração dos procedimentos clínicos, a construção dos POPs deu-se em três etapas.

A primeira consistiu no levantamento de todos os procedimentos operacionais necessários para garantir a qualidade da assistência prestada na Instituição. Nesse momento, foi necessário elencar todos os procedimentos de saúde, referentes a procedimentos de enfermagem, farmácia, nutrição, fisioterapia e atendimento ao público, realizados pelo Hospital e identificar quais destes não tinham POP.

Para cada procedimento realizado no Hospital é necessário a elaboração de um POP distinto no qual descreve-se a execução de uma atividade específica considerando os elementos de cada instituição de saúde, por exemplo, estrutura física (BARBOSA, 2011). Dessa forma, os procedimentos detectados foram divididos por área, por exemplo, clínica pediátrica, neurologia, cirurgia e subdivididos conforme procedimento.

A fase seguinte visou decidir os profissionais que seriam responsáveis pela elaboração e validação desses protocolos. Esses profissionais colaboradores da Instituição foram escolhidos conforme suas especialidades e funções desempenhadas na empresa. Para isso, é importante que a elaboração do POP seja efetuada por uma equipe que detenha o conhecimento, no caso, do técnico do procedimento e da unidade hospitalar na qual esse POP deverá ser efetuado (BARBOSA, 2011). Com isso, após a seleção desses profissionais eles dividiram-se em equipes e iniciaram a elaboração dos POPs.

Na última etapa foi finalmente realizada a discussão e validação de novos protocolos assistenciais. Para isso, semanalmente, cada equipe reuniu-se com todos os envolvidos na execução dos POPs e com os gestores do Hospital a fim de apresentar, esclarecer possíveis dúvidas, adequar, se necessário, e validar o procedimento.

Essas reuniões para a aprovação dos protocolos ocorreram para que fossem discutidas com mais afinco, todas as etapas para a construção dos procedimentos, garantindo profissionais experientes e especialistas na construção de cada fase do procedimento. Nesse momento, se necessário, faziam-se mudanças e adaptações propostas pela equipe e em seguida era validado.

A estratégia adotada para a elaboração dos procedimentos baseou-se na consulta de revisões de literatura científicas e tecnológicas, levando em consideração os aspectos políticos, sociais, econômicos, culturais e biológicos, culminando com a cultura de segurança do paciente da Instituição, a fim de garantir a qualidade dos serviços.

\section{RESULTADOS E DISCUSSÃO}

$\mathrm{Na}$ Instituição em questão, existiam alguns POPs referentes a processos decorrentes da prática em saúde. No entanto, o gerenciamento desses procedimentos tornou-se prioridade há quatro anos. Desde então, ações voltadas para a melhoria do exercício em saúde vinha se fortalecendo com o passar do tempo, objetivando a melhoria da qualidade da assistência.

Entretanto, muitos protocolos essenciais para a segurança do paciente, ainda necessitavam ser elaborados de acordo com a Portaria Ministerial número 529 de primeiro de Abril de 2013, lançada pelo Ministério da Saúde e pela Agência Nacional de Vigilância Sanitária, a qual institui o Programa Nacional de Segurança do paciente e ressalta a importância da criação e validação de protocolos, guias e manuais voltados à segurança do paciente em diferentes áreas tais como: Prevenção de infecções relacionadas à assistência à saúde; procedimentos cirúrgicos e de anestesiologia; prescrição, transcrição, dispensação e administração de medicamentos, sangue e hemoderivados; processos de identificação de pacientes; comunicação no ambiente dos serviços de saúde; prevenção de quedas; úlceras por pressão; transferência de pacientes entre pontos de cuidado; e uso seguro de equipamentos e materiais (BRASIL, 2013).

Dessa forma, atendendo às exigências do Ministério da Saúde e visando produzir, sistematizar e difundir conhecimentos, implantar a gestão de risco e fomentar a inclusão do tema segurança do paciente entre os colaboradores do hospital decidiu-se, em 2017, elaborar os POPs necessários para aprimorar o trabalho da Instituição.

No total foram criadas cinco equipes multiprofissionais, cada equipe ficou responsável pela elaboração de sete POPs. Os profissionais envolvidos no processo elaboraram 35 protocolos assistenciais à saúde, os quais abordavam procedimentos de enfermagem, farmácia, nutrição e fisioterapia, conforme mostra tabela 1 abaixo.

Quanto à enfermagem foram criados 27 (vinte e sete) POPs. Os temas abordados referem-se à assistência ao paciente crítico, cuidados com o paciente neurológico e assistência ao paciente pediátrico. Além desses, os POPs clínicos quanto aos cuidados com a gestante e recém nascido foram criteriosamente elaborados. Salienta-se que os protocolos acerca dos procedimentos necessários para garantir a segurança do paciente foram cuidadosamente elaborados com a participação ativa dos coordenadores do núcleo de segurança do paciente, coordenadora da comissão de controle de infecção hospitalar e os presidentes das comissões sentinelas farmacovigilância, hemovigilância e tecnovigilância existentes na Instituição.

Para a nutrição os 4 (quatro) protocolos elaborados abordaram o cuidado com o paciente que faz uso de dietas enterais, aperfeiçoamento do trabalho da equipe de profissionais que realizam ou orientam a ordenha de leite humano os quais formam o banco de leite materno e higienização do material utilizado no serviço de nutrição enteral.

O protocolo elaborado pela fisioterapia priorizou a assistência ao paciente que necessita de ventilação mecânica.

Os 3 (três) POPS elaborados pela farmácia envolveram os cuidados iniciais ao cliente durante a dispensação de medicamentos ao paciente internado, armazenamento adequado de medicamentos na central de abastecimento farmacêutico e a correta manipulação de medicamentos quimioterápicos. 
Tabela 1. Protocolos operacionais padrão elaborados pelos profissionais da Santa Casa de Misericórdia de Sobral, em 2017

Protocolos elaborados pela Nutrição:

Preparo de dietas enterais

Conservação do leite materno pós-ordenha

Higienização de materiais do serviço de nutrição enteral

Ordenha do leite humano

Protocolos elaborados pela Enfermagem:

Assistência de enfermagem ao recém-nascido em nutrição parenteral

Transporte intra-hospitalar de pacientes pediátricos não críticos

Salinização de cateter venoso periférico e acesso venoso centralpediátricos

Assistência de enfermagem ao paciente com síndrome de Guilliam-Barré

Identificação do paciente

Prevenção de lesão por pressão

Condutas de enfermagem diante as reações transfusionais imediatas

Assistência de enfermagem ao paciente com choque não hemorrágico

Admissão e assistência de enfermagem a gestante na cardiotocografia

Admissão e assistência de enfermagem em alojamento conjunto

Assistência de enfermagem a gestante com placenta prévia e descolamento prematuro de placenta

Assistência de enfermagem a gestante com pré-eclampsia e eclampsia

Assistência de enfermagem a gestante com ruptura prematura das membranas ovulares

Assistência de enfermagem ao paciente com transtorno degenerativo

Assistência de enfermagem ao paciente com traumatismo raquimedular

Assistência de enfermagem ao paciente portador de derivação ventricular externa

Assistência de enfermagem ao paciente submetido a angiografia cerebral

Assistência de enfermagem ao paciente vítima de traumatismo cranioencefálico

Atendimento de enfermagem ao paciente com trauma de tórax

Atendimento inicial de enfermagem a vítima de enforcamento

Atendimento inicial de enfermagem ao paciente com acidente vascular cerebral

Atendimento inicial de enfermagem ao paciente com intoxicação exógena

Atendimento inicial de enfermagem ao paciente com queimadura

Atendimento inicial de enfermagem ao paciente politraumatizado

Atendimento inicial de enfermagem ao paciente vítima de afogamento

Assistência de enfermagem ao paciente com choque hipovolêmico

Controle de drenagem gástrica

Protocolos elaborados pela Fisioterapia:

Assistência da fisioterapia aos pacientes submetidos a ventilação mecânica

Protocolos elaborados pela Farmácia:

Dispensação de medicamentos ao paciente internado

Armazenamento adequadode medicamentos na Central de Abastecimento Farmacêutico

Manipulação de agentes quimioterápicos

Ressalta-se que foram revisados e atualizados 169 procedimentos os quais faziam parte da rotina da Instituição. Todos os protocolos foram padronizados e apresentaram itens essenciais ao seu entendimento, entre eles, cabeçalho contendo o título, logotipo da instituição, número de páginas e datas da elaboração, aprovação e revisão, definição, objetivos, descrição detalhada do procedimento, abreviações, referências, anexos e responsáveis por sua validação.

A aprovação final do POP deu-se pela assinatura do diretor geral do Hospital, coordenador do núcleo de segurança do paciente, coordenadora da comissão de controle de infecção hospitalar e coordenador do Serviço Especializado em Engenharia de Segurança e em Medicina do Trabalho.

Após a conclusão de todos os protocolos, estes, foram disponibilizados aos seus usuários com acesso em papel, sob a responsabilidade do coordenador do setor e/ou em formato eletrônico, através da intranet para facilitar a consulta desse material pelos profissionais e acompanhar as próximas revisões e atualizações.

A elaboração de procedimentos é considerada uma importante estratégia, visto que a cultura de segurança do paciente em estabelecimentos de saúde é um passo importante para alcançar a qualidade na assistência em saúde, causando impacto direto na assistência.

Cabe ressaltar que a redução dos riscos e dos danos e a incorporação de boas práticas favorecem a efetividade dos cuidados em saúde e o seu gerenciamento de modo seguro e que trabalhar Procedimentos Operacionais Padrões é fundamental para atingir melhores indicadores (MILAGRES, 2015).

Atualmente, a padronização é considerada a mais fundamental das ferramentas gerenciais. Tais rotinas e procedimentos são considerados a base para uma organização segura (VINCENT; AMALBERTI, 2016).

Segundo Pimenta et al. (2015) há princípios estabelecidos para construção e validação de protocolos de assistência/cuidado, como a definição clara do foco, da 
população a que se destina, quem é o executor das ações, qual a estratégia de revisão da literatura e análise das evidências utilizadas. No entanto, há amplas evidências de que essas regras são habitualmente ignoradas pelos próprios profissionais de saúde (PEREIRA et al., 2010).

Conforme a experiência tem demonstrado, a situação brasileira no tocante à padronização não é boa. Falta literatura e faltam educação permanente e treinamento das pessoas que ocupam cargos de chefia. Nas raras empresas que se consideram padronizadas, este assunto tem sido relegado aos técnicos, apesar de ser um encargo essencialmente gerencial (HONÓRIO; CAETANO, 2009).

Sabe-se que as regras e os procedimentos nunca representam uma solução completa para a segurança. Porém, os protocolos são instrumentos legais, construídos dentro dos princípios da prática, baseada em evidências e oferecem as melhores opções disponíveis de cuidados padronizados e especificados, justamente porque essas tarefas são essenciais para a prestação de um cuidado seguro e de alta qualidade (VINCENT; AMALBERTI, 2016).

Os principais obstáculos encontrados para a conclusão de todo o processo foi à dificuldade de reunir, semanalmente, o grupo de profissionais e gestores do Hospital para assistir a apresentação do procedimento discussão e posterior validação do processo.

Entre os protocolos elaborados, estão o de acompanhamento de acidente com material biológico, coleta de sangue, administração de nutrição enteral, transporte intra-hospitalar de pacientes pediátricos e adultos não críticos, acolhimento com classificação de risco, admissão da gestante, parturiente e puérpera na maternidade, transporte de produtos estéreis para as unidades clínicas, paramentação cirúrgica, assistência de enfermagem ao paciente portador de derivação ventricular externa, sondagem gástrica no recém-nascido para gavagem e manipulação de medicamentos antineoplásicos.

A magnitude dessa problemática salienta a importância de não esperar por mudanças predeterminadas ou políticas específicas, e sim, desenvolver atitudes proativas em prol da segurança. As transformações começam a ter sentido e resultado a partir de mudanças na prática, as quais necessitam acontecer em curto prazo (PEDREIRA, 2006).

Esforços contínuos devem ser priorizados na prática, desde a alta direção, aos profissionais da assistência direta, com o intuito de promover estrutura física, humana e organizacional em qualidade e quantidade que garanta a promoção da cultura de segurança no hospital e a satisfação dos colaboradores, pacientes e familiares (OLIVEIRA, 2014).

No entanto, os profissionais de saúde e as pessoas que ocupam cargos gerenciais precisam entender que a padronização é o caminho mais seguro para a produtividade e competitividade em nível internacional, e constitui uma das bases sobre as quais se assenta o moderno gerenciamento. Tal investimento deve levar em consideração aspectos voltados para o gerenciamento com pessoas, remuneração adequada e estabelecimento de bom relacionamento interpessoal por meio de incentivo à comunicação efetiva e ao trabalho em equipe.

\section{CONCLUSÕES}

Merece destaque, as ações propostas relacionadas à correta identificação do paciente e implantação de medidas cabíveis para aumentar a adesão dos profissionais, quanto à higienização adequada das mãos.

No decorrer da construção dos POPs, percebeu-se uma mobilização entre os colaboradores da Instituição que possibilitou a identificação dos riscos e dos danos e a incorporação de boas práticas dos cuidados em saúde. Esta melhoria dependeu apenas da necessária mudança de cultura dos profissionais para a segurança, do uso de indicadores de qualidade, da existência de um sistema de registros, alinhados à política de segurança do paciente instituída nacionalmente.

Após a elaboração dos protocolos padrão tem-se como desafio implantá-los em todo o hospital. Para isso, devem-se realizar treinamentos permanentes para todos os colaboradores a fim de garantir a qualidade $e$ a uniformidade de todos os processos envolvidos na condução de estudos.

\section{REFERÊNCIAS}

ARANAZ-ANDRÉS, J.; AIBAR-REMÓN, C.; LIMÓNRAMÍREZ, R.; AMARILLA, A.; RESTREPO, F. R.; URROZ, O.; SARABIA, O.; GARCÍA-CORCUERA, L. V.; TEROL-GARCÍA, E.; AGRA-VARELA, Y.; GONSETH-GARCÍA, J.; BATES, D. W.; LARIZGOITIA, I. Prevalenceof adverse events in thehospitalsoffiveLatin American countries: results of the 'Ibero american study of adverse events'(IBEAS). BMJ Quality \& Safety, vol. 20, n. 12, pag. 1043-1051, 2011.

BARBOSA, C. M.; MAURO, M. F. Z.; CRISTOVÃO, S. A. B.; MANGIONE, J. A. A importância dos procedimentos operacionais padrão (POPs) para os centros de pesquisa clínica. Revista da Associação Médica Brasileira. vol. 57, n. 2, pag: 134-135. 2011.

BRASIL. Ministério da Saúde. Portaria no . 529, de 1 de abril de 2013. Institui o Programa Nacional de Segurança do Paciente (PNSP). Diário Oficial da União, Brasília, 1 de abril de 2013. Seção I.

BROZEK, J. L.; AKL, E. A.; ALONSO-COELLO, P.; LANG, D.; JAESCHKE, R.; WILLIAMS, J. W.; PHILLIPS, B.; LELGEMANN, M.; LETHABY, A.; BOUSQUET, J.; GUYATT, G. H.; SCHÜNEMANN, H. J. Grading quality of evidence and strength of recommendations in clinical practice guidelines. Part 1 of 3. An overview of the GRADE approach and grading quality of evidence about interventions. Allergy, vol. 64, n. 5, pag: 669- 677, 2009.

DAINESI, S. M.; NUNES, D. B. Procedimentos operacionais padronizados e o gerenciamento de qualidade em centros de pesquisa. Rev. Assoc. Med. Bras., São Paulo, v. 53, n. 1, p. 6, 2007.

HONÓRIO, R. P. P.; CAETANO, J. A.; Elaboração de um protocolo de assistência de enfermagem ao paciente 
hematológico: relato de experiência. Rev. Eletr. Enf.; vol. 11, n. 1 pag: 188- 193. 2009.

JOUVETL, P.; HERNET, P.; WYSOCKI, M. Development and implementation of explicit computerized protocols for mechanical ventilation in children. Ann Intensive Care.Volume: 01, numero: 01, pagina: 51, 2011.CHARLES, V. Segurança do paciente: orientações para evitar os eventos adversos. Porto Alegre (RS): Yendis; 2010.

MENDES, W.; MARTINS, M.; ROZENFELD, S.; TRAVASSOS, C. The assessmentof adverse events in hospitals in Brazil.InternationalJournal for Quality in Health Care, v. 21, n. 4, p. 279-284, 2009.

MILAGRES, L. M. Gestão de riscos para segurança do paciente: o enfermeiro e a notificação dos eventos adversos. 2015. 100 folhas. Dissertação (Mestrado em Enfermagem). Faculdade de Enfermagem, Universidade Federal de Juiz de Fora. Juiz de Fora - Minas Gerais. 2015 .

OLIVEIRA, R. M.; LEITÃO, I. M. T. A.; SILVA, L. M. S.; FIGUEIREDO, S. V.; SAMPAIO, R. L.; GONDIM, M. M. Estratégias para promover segurança do paciente: da identificação dos riscos às práticas baseadas em evidências. Esc. Anna Nery, Rio de Janeiro, v. 18, n. 1, p. 122-129, 2014

PEDREIRA, M. L. G.; Errar é humano: estratégias para a busca da segurança do paciente. In: HARADA, M. J. C. S;
PEDREIRA, M. L. G.; PETERLI, M. A. S.; PEREIRA, S. R.; organizadores. O erro humano e a segurança do paciente. São Paulo: Atheneu, 2006.

PEREIRA, L. L.; CHANES, M.; GALVÃO, C. R.; Gestão em saúde: tendências, inovações e perspectivas. São Paulo: Centro Universitário São Camilo, paginas: 458459. 2010.

PIMENTA, C. A. M.; PASTANA, I. C. A. S. S.; SICHIERI, K.; SOLHA, R. K. T.; SOUZA, W. Guia para construção de protocolos assistenciais de enfermagem; COREN-SP, São Paulo: COREN-SP, 2015.

SILVA, L. D; CAMERINI, F. G. Análise da administração de medicamentos intravenosos em hospital da rede sentinela. Texto contexto - enfermagem, Florianópolis ,v. 21,n. 3,p. 633-641, Setembro. 2012.

VINCENT, C.; AMALBERTI, R.; Cuidado de Saúde mais Seguro: estratégias para o cotidiano do cuidado / Charles Vincent e Rene Almaberti - Rio de Janeiro, 2016.

WACHTER, R. Compreendendo a Segurança do Paciente. $2^{a}$ Ed. Porto Alegre: AMGH, 2013. 478p.

WERNECK, M. A. F.; Protocolos de cuidados à saúde e de organização do serviço/ Marcos Azevedo Furkim Werneck, Horácio Pereira de Faria e Kátia Ferreira Costa Campos. Belo Horizonte: Nescon/UFMG, Coopmed, 2009. 90 p. 